\title{
Sakhalin real-time shaking map tool for industrial and civil facilities safety
}

\author{
Andrey Stepnov ${ }^{1}$, Alexey Konovalov ${ }^{1, *}$, Vitaliy Klachkov ${ }^{1}$, Roman Dmitrienko ${ }^{1}$, Alexander \\ Gavrilov $^{1}$, Evgeny Bogdanov ${ }^{1}$ and Konstantin Manaychev ${ }^{1}$ \\ ${ }^{1}$ Geophysical Technologies LLC, 116 Purkaeva St, Yuzhno-Sakhalinsk, 693006, Russia
}

\begin{abstract}
Regional shaking map tool for rapid characterization of the strong ground motion following the significant earthquake has been developed. New tool is based on state-of-the-art data processing technologies and regional seismological data. The earthquake data processing system uses regional and global seismic stations. The technologies for generating shaking maps is mostly automatic. Public information on recent and past earthquakes is freely available on the eqalert.ru website. Internet users can also submit the felt report through the eqalert.ru using "Did you feel the earthquake?" questioner, unified in accordance with the U.S. Geological Survey DYFI. Automatic earthquake notification is freely available in the form of PUSH-notification for Android and iOS mobile applications and Telegram messenger bot.
\end{abstract}

\section{Introduction}

The main goal of shaking map is to produce near-real time maps of ground shaking (peak ground acceleration, response spectra, seismic intensity etc.) following significant earthquakes. Commonly the results are rapidly available via the Worldwide Web through a variety of map formats. Shaking map produces a graded distribution of ground motion at sites based on distance from the earthquake source, earthquake magnitude, soil conditions at sites and ground motion prediction equation (GMPE). The most popular shaking map product is ShakeMap [1], developed by U.S. Geological Survey. It facilitates communication of earthquake information beyond just magnitude and location of the source.

So, shaking map is a tool for emergency response, public information, loss estimation and post-earthquake engineering and scientific analyses. It is actively used by the government, emergency surveys, industrial companies and scientific community.

The main goal of our research is to develop the regional shaking map product (EQA!ert Project [2]) based on new data processing technologies, state-of-art methods of earthquake notification, regional earthquake information and recent geological data.

\footnotetext{
*Corresponding author: a.konovalov@geophystech.ru
} 


\section{Tectonic Settings}

A boundary between the Eurasian (Amurian) and the Okhotsk (North American) plate is supposed to run from north to south of Sakhalin Island. Seismicity is the primary basis for identifying the plate boundary and tectonic setting of the region. Sakhalin Island is dominated structurally by compressive features. Significant earthquakes with magnitude $M \geq 7$ occurred approximately once per 10 years in the Sakhalin-Japanese seismogenic zone [3].

According to the geological data the slip rate associated with the regional thrust faults is about 1-3 mm per year [4]. GPS observation indicates the same order of horizontal velocity of surface deformation. On 27 May, 1995 a catastrophic earthquake (Mw=7.1) occurred in the northern part of Sakhalin Island. More than seventeen civil buildings were destroyed in the town Neftegorsk, and more than 2000 people were died. Shaking map was not available in 1995. Dramatically, communication infrastructure was destroyed by serve ground shaking. Initial instrumental epicentre was located far enough from town as reported by regional seismological agency. In total for many hours the government and emergency surveys had been underestimated the level of ground shaking and loss parameters.

\section{EQA!ert Project}

\subsection{Seismic network}

The existing seismic network of EQA!ert Project consists of 17 stations (Fig. 1) that cover almost whole area of Sakhalin Island and its offshore zone. Seismic stations continuously transmit seismic recordings to the earthquake data processing system in the near real-time mode. Stations are coupled into a single VPN network based on GPRS/HSDPA, XDSL, and IPSEC technologies [5].

ARGI, NGLK, CHIVO, and TUN stations (Fig. 1) are equipped with short-period LE3Dlite MKII seismometers (Lennartz-Electronic, Germany) and Delta-03 recorders (LogiS LLC, Russia).

NYSH and LNSK are equipped with SIXAOLA 2 v3 seismic station (OSOP, Republic of Panama). The equipment consist of short-period seismometer with a natural frequency of $0.5 \mathrm{~Hz}$, MEMS-type accelerometer (recording range $\pm 2 \mathrm{~g}$ ), and a built-in recorder that supports the SEEDLINK protocol.

SMY, ILY, and SYN are equipped with Raspberry Shake 4D seismic station (OSOP, Republic of Panama). The equipment consist of short-period seismometer with a natural frequency of $1 \mathrm{~Hz}$, MEMS-type accelerometer (recording range $\pm 2 \mathrm{~g}$ ), and a built-in recorder that supports the SEEDLINK protocol.

In order of improving the accuracy of automatic earthquake source location the system is integrated with regional seismic stations of the Far Eastern Branch of the Russian Academy of Sciences (Fig. 1). A732 (BB8F) regional station is equipped with broad-band REFTEK 151/RETEK 130 seismometers with a natural period of $120 \mathrm{sec}$ (Trimble, United States of America) and operating in the real-time mode via the RTPD protocol. The regional seismic network is complemented with a broadband stations YSS, ASAJ (Streckeisen STS-1/VBB) of GSN - IRIS/USGS. 


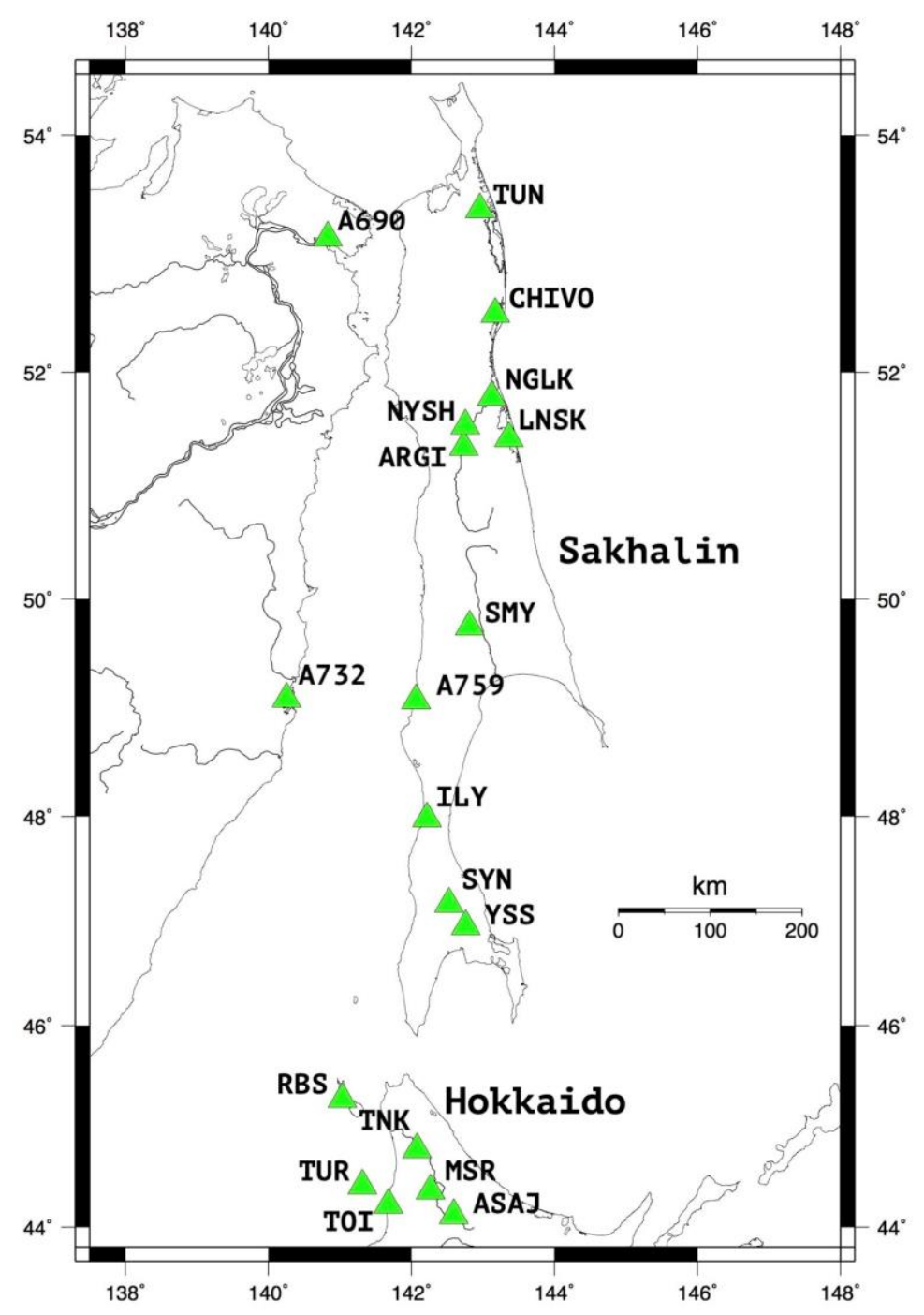

Fig. 1. Seismic network of EQA!ert Project.

The system also collects the real time records from short-period seismometers located in the north of Hokkaido Island (Institute of Seismology and Volcanology of Hokkaido University, Japan): RBS, TNK, TUR, TOI, MSR. These stations are equipped with shortperiod seismometers with a natural frequency of $1 \mathrm{~Hz}$. Data of Hokkaido University's seismic network are integrated using the WIN software package [6], RINGSERVER, and our application for converting data from the WIN format to the MINISEED format.

\subsection{Earthquake data processing}

The waveforms has been processed in two steps. At the first step the standard STA/LTA arrival detection algorithm has been used, and source parameters of significant earthquakes automatically processed by the EARTHWORM software [7] (Fig. 2). Source parameters are automatically reported at the EQA! ert web-site [2] within several ( 3) minutes after the origin time. 


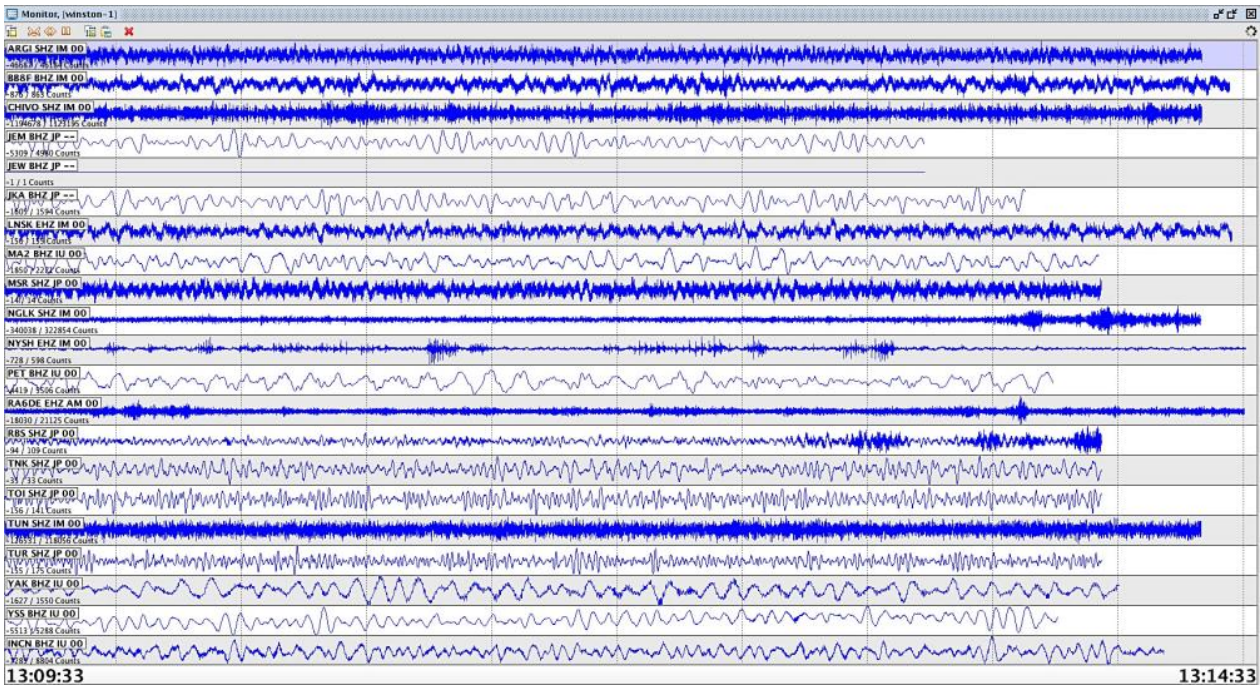

Fig. 2. Real-time EARTHWORM monitor of EQA!ert Project.

At the second step SEISAN software [8] is applied in order to check the automatic picks, location and magnitude. The weak seismicity has been manually processed by SEISAN software.

The computer cluster based on the EARTHWORM and SEISAN (OS GNU \Linux) software functions on the hardware and information telecommunication resources of the Data Centre of the Far Eastern Branch of the Russian Academy of Sciences [9].

The velocity structure, magnitude, equipment calibration parameters and software configuration of existing earthquake data processing system is given in [10-13].

The results of earthquake source location, shaking map and moment tensor solutions are reported at the web-site https://eqalert.ru/ru.

\subsection{Stack of technologies}

The EQA!ert Project uses state-of-art information technologies (IT). Its architecture is built on microservice patterns. The IT stack consists of the following components:

1. API: the centralized orchestration system. API receives from and responses to its clients, sends requests to and get responses from external systems: databases, seismic stations, processing modules, etc. This component is written with PHP v7 programming language using Laravel web framework.

2. Clients. Browsers, mobile applications, external websites, CLI applications, and other application interacting with the HTTP/1.1 protocol formally appears to clients. Clients can get the requested data in such formats as HTML, JSON, and XML. We are going to add more formats. The official website eqalert.ru is a single page application which is written with JavaScript web framework Vue.js.

3. Databases. The EQA! ert Project uses MariaDB relational database management system for storing data transmitted from processing modules, clients, external websites etc.

4. Processing modules. These modules receive waveforms and post-processed seismic data and make the further data processing (magnitude determination, source location, shaking map producing etc.). The results are transmitted back to API. These modules are built with the high-performance language Julia.

5. Seismic stations. Stations collect, and send seismic data in the near real-time mode to the earthquake data processing system. The system processes the data and sends it to API for 
further post processing and storing. This data will be sent to the clients as soon as it gets processed. All the components are run on Linux operating systems with Long Term Support life cycle.

\subsection{Sakhalin GMPE}

We have developed the regional GMPE based on Morikawa and Fujiwara [14] formulation:

$$
\log A=a_{2} M w_{2}^{\prime}+b_{2 \_} X+c_{2 \_k}-\log \left(X+d_{2} 10^{e_{2} M w_{2}^{\prime}}\right) \pm \sigma_{2}
$$

where

$A-\mathrm{PGA}(\mathrm{cm} / \mathrm{sec} / \mathrm{sec})$;

$a_{2}, b_{2 \_}, c_{2 \_}, d_{2}, e_{2}-$ constants;

$k$ denotes the earthquake type: 1 - crustal, 2 - interplate, 3 - intraplate;

$X$ - closet distance to the rupture plane $(\mathrm{km})$;

$\sigma_{2}$ - standard deviation;

$M w_{2}^{\prime}=\min \left(M w, M w_{02}\right)$.

Best fitting of regional PGA data gives the constant $a_{2}, b_{2 \_}, c_{2-k}, d_{2}, e_{2}$. The moment magnitude is calculated by the regional ML-Mw relation. $M w_{02}$ is taken 6.5.

The site correction term is given by:

$$
\log A_{G s}=\log A+G s
$$

where

$A_{G s}$ - site corrected peak ground acceleration;

$G s=p_{s} \log \left[\min \left(V_{s \max }, V_{s 30}\right) / V_{0}\right]$;

$p_{s}, V_{s \text { max }}, V_{0}-$ constants [14];

$V_{s 30}$ - average shear-wave velocity down to $30 \mathrm{~m}$.

\subsection{Soil effects}

In order to make a site correction we use the seismic shear wave velocities in the upper 30$\mathrm{m}$ ground layer (Vs30) according to the topographic slope for Sakhalin Island from USGS Global Vs30 Map Server [15]. At the moment we have collected the database of Vs30 in various sites of Sakhalin Island according to the seismic hazard studying and microzonation services. The data will be used in the near future for developing the regional regression between Vs30 and topographic slope.

\subsection{Online felt reports}

Internet users can also submit the felt report through the eqalert.ru "Did you feel the earthquake?" questionary. The U.S. Geological Survey DYDI questionary translated into Russian was used for processing the intensity data. The felt reports of the respondents from each settlement was transformed to Community Weighted Sum (CWS) with the calculation of the Community Decimal Intensity (CDI) using equation $[16,17]$ :

$$
\mathrm{CDI}=3.4 \ln (\mathrm{CWS})-4.38 \text {. }
$$

The obtained CDI values were rounded to the first number after the comma. In general, CDI values should be similar to the MMI values [16].

At the moment more than 300 felt reports are available. It is very important tool for the emergency response. 


\subsection{Earthquake notification}

At the moment several ways of earthquake notification are implemented in the EQA!ert Project. Users may choose the most preferable way for getting up-to-date earthquake information without visiting the eqalert.ru website. The Telegram messenger bot is also used as tool for rapid notification. Optionally it displays lists of recent seismic events.

PUSH-notifications are available for mobile EQA!ert applications for Android and iOS. Additionally users may receive earthquake notifications and a brief seismic reports by email.

\subsection{Shaking map examples}

A reverse fault earthquake with the moment magnitude $\mathrm{Mw}=5.8$ occurred in the middle part of the Sakhalin Island on 14 August, 2016. The earthquake source was related to the Central Sakhalin Fault Zone, which is considered to mark the boundary between the Okhotsk and Eurasian plates [18]. The peak ground acceleration (PGA) map is shown in Fig. 4. The PGA value about $0.12 \mathrm{~g}$ was measured by strong motion station at the epicentral distance $19 \mathrm{~km}$. The shaking map was available within several minutes after mainshock origin. Within hours of the mainshock more than 100 felt reports were received. Maximum intensity $\mathrm{MMI}=6.2$ was reported at the settlement Onor (distance $27 \mathrm{~km}$ ). Another example is associated with southern part of Sakhalin Island. The examined earthquake occurred on 7 April, 2017. The local magnitude was $\mathrm{ML}=4.1$. Seismic event was felt in the nearest settlements (up to MMI=4). The maximum recorded PGA value was $0.016 \mathrm{~g}$ at epicentral distance $6 \mathrm{~km}$.

As a final example a small seismic event $(\mathrm{ML}=3.8)$ is considered. The automatic earthquake data processing system missed this event. Seismic recordings were processed manually and earthquake location and magnitude was determined 1 hour after the earthquake origin. The shaking map become available immediately after updating earthquake data.

It should be noted that there was no official responses and earthquake reports from Russian emergency survey and seismological agencies. Regional mass media [21, 22] reported about light to moderate ground shaking in Uglegorsk and Shakhtersk. So, the EQA!ert shaking map was the only public resource given the earthquake information.

\section{Conclusion}

We have developed regional shaking map tool for rapidly characterizing the parameters of strong ground shaking (peak ground acceleration, response spectra, seismic intensity etc.) following the significant earthquake. Regional ground motion prediction equation is the basis for producing the peak acceleration and seismic intensity maps.

The earthquake data processing system uses regional and global seismic station in order to improve the accuracy of source location and data processing time delay. The technologies for generating shaking maps is mostly automatic. Public information (source location, shaking map, moment tensors etc.) on recent and historical earthquakes is available on the eqalert.ru website. EQA! ert products and data are freely open to the global community.

Internet users can also submit the felt report through the eqalert.ru "Did you feel the earthquake?" questionary, unified in accordance with the U.S. Geological Survey DYFI.

Automatic earthquake notification is freely available in the form of PUSH-notification for Android and iOS mobile applications and Telegram messenger bot.

\section{References}

1. URL : https://earthquake.usgs.gov/data/shakemap/ 
2. URL: https://eqalert.ru/ru

3. K. Katsumata, M. Kasahara, M. Ichiyanagi, Bul. Seis. Soc. Am. 94-1, 117-130 (2004)

4. A.I. Kozhurin, Active Geodynamics of North-Western Sector of the Pacific Ocean Tectonic Belt (based on the data of studying the active fractures), Author's abstract of thesis for the degree of Doctor of Geological and Mineralogical Sciences (Moscow, 2013)

5. A.A. Stepnov, A.V. Konovalov, A.V. Gavrilov, K.A. Manaychev, Seism Instrum 53(4), 267-279 (2017) doi.org/10.3103/S0747923917040107

6. T. Urabe, S. Tsukuda, Progr. Abstr., Seismol. Soc. Jpn. 2, 41 (1992)

7. URL: http://www.earthwormcentral.org

8. J. Havskov, L. Ottemoller, Seismol. Res. Lett. 70(5), 532-534 (1999)

9. A.A. Sorokin, S.P. Korolev, A.N. Polyakov, Procedia Computer Science 66, 584-591 (2015)

10. A.A. Stepnov, A.V. Gavrilov, A.V. Konovalov, L. Ottemöller, Seism. Instrum. 50-1, 6774 (2014)

11. A.V. Konovalov, A.A. Stepnov, V.N. Patrikeev, Seism. Instrum. 48-3, 270-281 (2012)

12. A.V. Konovalov, A.S. Sychev, J. Volcanol. Seismol. 8-6, 390-400 (2014)

13. N. Morikawa, H. Fujiwara, Journal of Disaster Research 8-5 (2013)

14. http://earthquake.usgs.gov/hazards/apps/vs30/

15. L.A. Dengler, J.W. Dewey, Bull. Seism. Soc. Am. 88(2), 441-462 (1998)

16. D.J. Wald, V. Quitoriano, C.B. Worden, M. Hopper, J.W. Dewey, Annals of Geophysics 54(6), 688-709 (2011)

17. A.V. Konovalov, A.A. Stepnov, D.A. Safonov, J. Seismol (2018) doi.org/10.1007/s10950-018-9744-y

18. URL: https://eqalert.ru/ru/events/QgpAn7OW

19. URL: https://eqalert.ru/ru/events/xEwjZBMy

20. URL: http://uglegorsk.news/zhiteli-uglegorska-i-shahtyorska-oshhutili-podzemnyiytolchok/

21. URL: https://sakhalin.info/news/138562

22. URL: https://eqalert.ru/ru/events/KEXKR1M0 